\title{
Frequency conversion of mid-infrared optical signals into the telecom band using nonlinear silicon nanophotonic wires
}

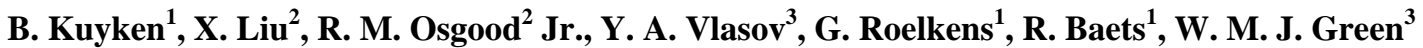 \\ ${ }^{1}$ Photonics Research Group, Department of Information Technology, Ghent University - imec, Ghent B-9000, Belgium \\ ${ }^{2}$ Microelectronics Sciences Laboratories, Columbia University, New York, NY 10027, USA. \\ ${ }^{3}$ IBM T. J. Watson Research Center, 1101 Kitchawan Road, Yorktown Heights, NY 10598, USA. \\ Tel.:+32 (0)9 32-9-264-3335 . Email: Bart.Kuyken@intec.ugent.be
}

\begin{abstract}
We demonstrate parametric conversion with simultaneous 19.5dB amplification, of a mid-IR 2440nm signal to the telecom-band near $1620 \mathrm{~nm}$, using silicon nanophotonic wires. Conversion over $820 \mathrm{~nm}$ is facilitated using higher-order waveguide dispersion.

(C) 2010 Optical Society of America

OCIS codes: Integrated optics: 130.7405 Wavelength conversion devices, Integrated optics: 130.4310 Nonlinear.
\end{abstract}

\section{Introduction}

Nonlinear optical functions such as wavelength conversion and parametric amplification have been thoroughly investigated in single-mode [1] and photonic crystal fibers [2], as well as in chalcogenide [3] and silicon-oninsulator (SOI) planar integrated waveguide circuits [4]. The SOI platform is particularly appealing for four-wave mixing (FWM) based optical signal processing, as tight optical confinement in silicon nanophotonic wire waveguides gives rise to extremely high nonlinear parameters [5], enabling ultra-compact low-power devices. Moreover, the strong optical confinement permits application of waveguide dispersion engineering techniques to facilitate the required phase matching of the interacting signal, idler, and pump waves.

While much of the literature on silicon nanophotonic wire nonlinear devices has utilized a telecom-band pump [4-5], recent reports of wavelength conversion [6] and large on-chip/off-chip parametric amplification [7] have been made, using a mid-infrared (mid-IR) pump beam near one-half the band gap wavelength in order to avoid the parasitic effects of two-photon nonlinear absorption. These recent demonstrations raise the interesting prospect of developing a SOI photonics platform for mid-IR optical applications [8] including free-space communication, medical diagnostics, and imaging. One particularly attractive application is the possibility of receiving weak signals modulated onto a mid-IR optical carrier, performing ultra-broadband parametric wavelength conversion with gain via four-wave mixing, and finally detecting the signal using high-sensitivity telecom-band optical receivers. These receivers can be based upon established commercially-available III-V semiconductor technologies (i.e. InGaAs), or silicon nanophotonic wire-integrated detectors fabricated via heterogeneous (i.e. III-V) or monolithic (i.e. Ge, SiGe) approaches. Using such receivers to process a wavelength-converted mid-IR signal could potentially offer a large sensitivity enhancement over traditional direct mid-IR detection approaches based upon narrow band-gap semiconductors such as $\mathrm{HgCdTe}$ or GaSb, and could also alleviate the need for detector temperature control.

In this paper we exploit the higher-order waveguide dispersion terms to achieve phase matching in discrete bands widely separated from the pump, which allows for frequency conversion of optical signals from the mid-IR into the telecom wavelength range. We achieve conversion efficiencies and amplification up to $19.5 \mathrm{~dB}$ and $18.8 \mathrm{~dB}$ respectively, using a pump wavelength of $1946 \mathrm{~nm}$ with a peak power of $37.3 \mathrm{~W}$ in the waveguide.

\section{Phase matching using higher order dispersion terms}

Typically, the phase matching behavior near the pump wavelength for a four-wave mixing process can be approximated by using the waveguide dispersion information up-to second order. However in certain cases when parametric and frequency conversion gain band can extend to wavelengths far from that of the pump (i.e. conversion of mid-IR signals to the telecom band), taking higher-order dispersion terms into account becomes necessary. Mathematically taking the dispersion up to the fourth order in account, the equation governing the phase matching condition $\left(\Delta k_{\text {linear }}+\Delta k_{\text {nonlinear }}=0\right)$ becomes

$$
\beta_{2} \cdot(\Delta \omega)^{2}+\frac{1}{12} \beta_{4} \cdot(\Delta \omega)^{4}+2 \gamma P=0
$$

where $\beta_{2}, \beta_{4}$ are the second and fourth order dispersion coefficients, $\Delta \omega$ is the frequency detuning between the signal and the pump, $\gamma$ is the effective nonlinearity parameter, and $P$ is the peak power of the pump in the photonic wire. When the detuning is small, for example when working in a region near the pump, the fourth order dispersion 
term can be neglected and we can conclude that anomalous dispersion with negative $\beta_{2}$ leads to phase matching. Indeed parametric gain can be achieved in a broadband region around the pump by pumping silicon nanophotonic wires in the anomalous dispersion regime. However when the detuning becomes substantially large the higher-order term starts to dominate. Analyzing the above formula reveals that under the condition $\beta_{2}, \beta_{4}$ having opposite signs a second phase matching point arises where the linear and nonlinear phase mismatch cancel and parametric gain becomes possible in a discrete narrow band at these points. These discrete phase matching bands have been used to achieve amplification in fiber optical parametric amplifiers which are pumped in the normal dispersion regime [2], where the fiber exhibits negative fourth-order dispersion.
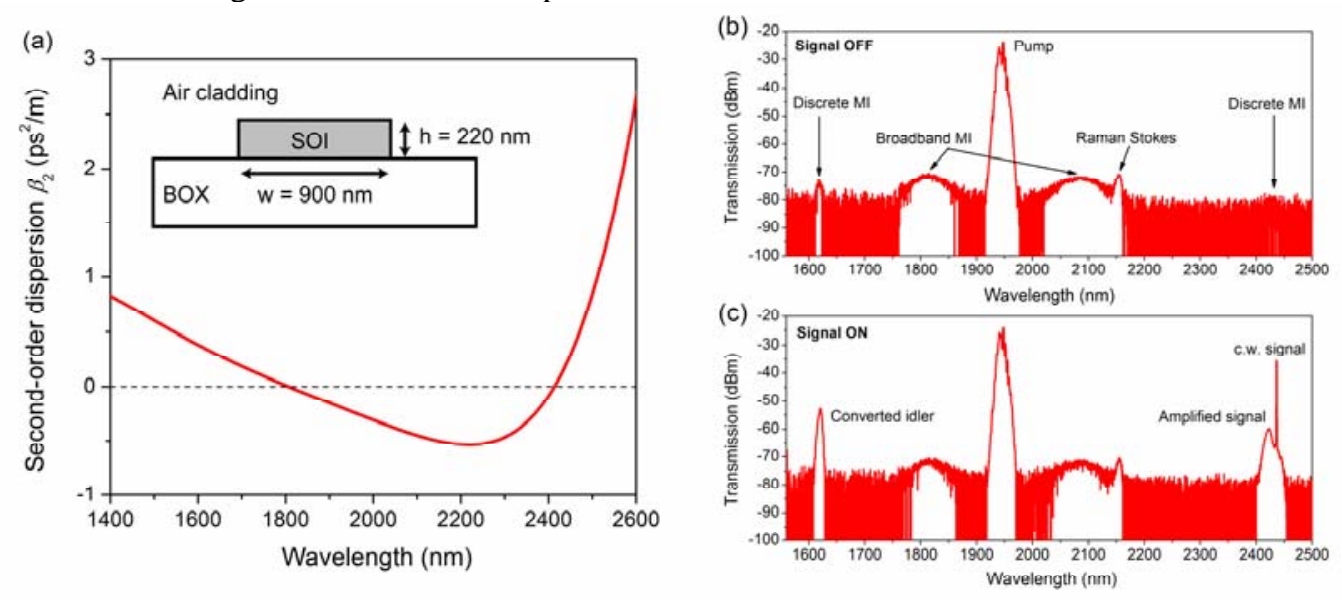

Fig. 1. (a) The second-order dispersion for the fundamental TE mode of the photonic wire. The second-order dispersion is anomalous $\left(\beta_{2}<0\right)$ between $1800 \mathrm{~nm}$ and $2410 \mathrm{~nm}$, and has positive curvature $\left(\beta_{4}>0\right)$ throughout the entire plotted range. Inset: Schematic of the SOI nanophotonic waveguide dimensions. (b) Signal off: Modulation instability output spectrum generated by the exponential amplification of noise in the sidebands when pumping at $1946 \mathrm{~nm}$. (c) Signal on: Parametric amplification of a continuous wave mid-IR signal at $2440 \mathrm{~nm}$, and simultaneous conversion to a wavelength of $1620 \mathrm{~nm}$.

\section{Experimental results}

The nanophotonic wires used were fabricated in a CMOS pilot line on $200 \mathrm{~mm}$ SOI wafers, consisting of a $220 \mathrm{~nm}$ silicon waveguide layer on a $2 \mu \mathrm{m}$ buried oxide layer. The wires were $900 \mathrm{~nm}$ wide with no top cladding, as shown in the inset of Figure 1. The high optical confinement results not only in a high effective nonlinearity parameter, but also gives rise to anomalous waveguide dispersion in the mid-infrared. The dispersion was simulated using a commercial finite element solver (RSoft FemSIM). Figure 1(a) illustrates the wire's anomalous dispersion $\left(\beta_{2}<0\right)$ for the TE polarized mode between the two zero dispersion wavelengths of $1810 \mathrm{~nm}$ and $2410 \mathrm{~nm}$, and has positive fourth order dispersion due to the positive curvature of the dispersion graph. Therefore, the opposing signs of $\beta_{2}$ and $\beta_{4}$ should permit discrete phase matching when this photonic wire is pumped within the range $1810 \mathrm{~nm}-2410 \mathrm{~nm}$.

For the experiment, a picosecond pulse train (FWHM $\sim 2 \mathrm{ps,} \mathrm{repetition} \mathrm{rate}=76 \mathrm{MHz}$ ) generated by a tunable optical parametric oscillator (OPO) coupled to a regular single-mode optical fiber is used as the four-wave-mixing pump. A continuous wave tunable mid-infrared laser is used as the signal. The pump and signal are multiplexed into a single fiber before coupling into a $2 \mathrm{~cm}$-long silicon nanophotonic wire. Coupling into/out of the SOI photonic wire is accomplished using lensed fibers. Polarization controllers are used to couple a TE polarized pump and signal to the photonic wire. The waveguide loss was found to be $2.5 \mathrm{~dB} / \mathrm{cm}$ for the TE mode, within both the telecom and the mid infrared bands of interest. The wavelength-dependent fiber coupling loss at each facet varied between $9 \mathrm{~dB}$ to $11 \mathrm{~dB}$. The output is characterized with a mid-infrared optical spectrum analyzer, at $1 \mathrm{~nm}$ spectral resolution.

Figure 1(b) and 1(c) shows representative output spectra obtained with the pump pulse train located at $1946 \mathrm{~nm}$, with peak power of $37.3 \mathrm{~W}$ at the input of the nanophotonic wire. In Figure 1(b) only the pump propagates through the silicon wire. The broadband amplification of noise due to modulation instability near the pump is clearly seen, in addition to a peak from the Raman Stokes-shifted pump at $2155 \mathrm{~nm}$. Moreover, an additional two discrete bands originating from higher-order phase matching are also visible in the modulation instability spectrum, located at 1620 $\mathrm{nm}$ and at $2440 \mathrm{~nm}$. The output power in the $2440 \mathrm{~nm}$ peak is reduced due to higher input/output waveguide coupling losses at longer wavelengths. Figure 1(c) shows the spectrum when the discrete bands are probed by a CW mid-IR laser at $2440 \mathrm{~nm}$. When the signal is tuned into this spectral band, it experiences exponential parametric gain, and is simultaneously wavelength converted to a telecom-band idler at $1620 \mathrm{~nm}$. In addition, the signal and converted idler are broadened by cross-phase modulation due to the pulsed nature of the source. 
By recording a series of similar spectra at different signal wavelengths, the parametric amplification and conversion efficiency can be determined as a function of wavelength within the discrete phase-matching bands [7]. This data is illustrated in Figure 2(a), indicating a peak net on-chip signal amplification of $18.8 \mathrm{~dB}$ and a conversion efficiency of $19.5 \mathrm{~dB}$. The on-chip gain exceeds $10 \mathrm{~dB}$ over a bandwidth of $10 \mathrm{~nm}$ near the idler $(1620 \mathrm{~nm})$, and a bandwidth of $62 \mathrm{~nm}$ near the signal $(2440 \mathrm{~nm})$. The large values of idler conversion gain indicate that silicon nanophotonic wires can potentially allow for efficient amplification of weak mid-IR signals, and subsequent detection using commercially available high-sensitivity telecom receivers.

Figure 2(b) shows the location of the discrete bands as a function of pump wavelength (defined as the modulation instability peak wavelength). Tuning the pump wavelength varies the dispersion accumulated along the silicon nanophotonic wire, and results in a spectral shift of the discrete bands where phase matching is obtained. The spectral separation of the bands becomes larger with increasing $\left|\beta_{2}\right|$ and decreasing $\left|\beta_{4}\right|$. The data illustrates that the discrete bands move closer to the pump wavelength when the pump approaches the zero dispersion wavelengths at $1810 \mathrm{~nm}$ and $2410 \mathrm{~nm}$, i.e. when $\beta_{2}$ decreases. Through further optimization of the wire dimensions so as to increase the second-order dispersion while simultaneously decreasing the fourth-order dispersion, it is possible to design a silicon wire capable of converting mid-IR signals directly to the C-band near $1550 \mathrm{~nm}$.
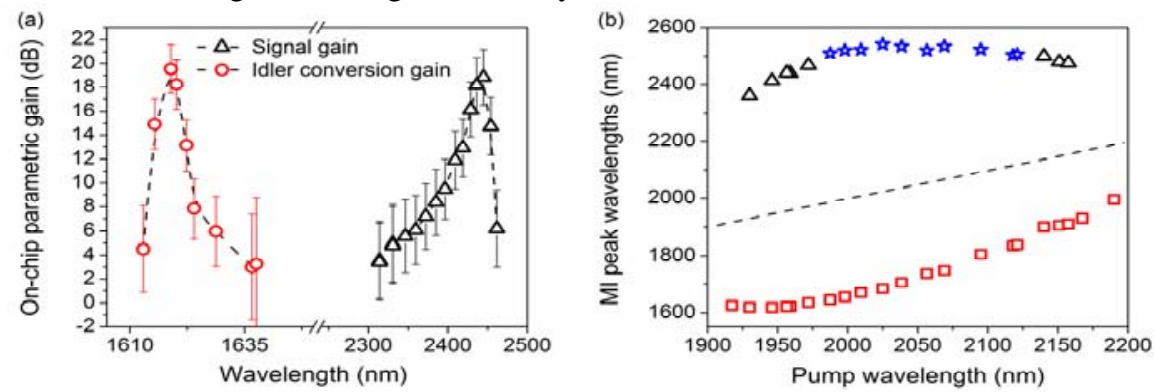

Fig. 2. (a) On-chip parametric signal gain and idler frequency conversion gain for the mid-infrared signals. Dashed lines are spline curves included to guide the eye. (b) Peak of the discrete modulation instability bands as a function of pump wavelength. The MI peaks indicated by the

blue stars was not directly measurable, as they were located beyond the $2500 \mathrm{~nm}$ maximum wavelength limit of the OSA used. Rather, the positions of these peaks were inferred from energy conservation. The dashed line tracks the position of the pump.

\section{Conclusion}

We have demonstrated the use of higher-order phase matching in silicon nanophotonic wires to realize the conversion of signals from the mid-IR into the telecommunication band, with simultaneous signal amplification of $18.8 \mathrm{~dB}$ and conversion gain of $19.5 \mathrm{~dB}$. These results can be applied to the processing of weak mid-IR signals using established high-sensitivity telecom-band optical receiver technology, potentially offering a large sensitivity enhancement over traditional direct mid-IR detection approaches using narrow band-gap semiconductors.

B.K., G.R. acknowledge the Flemish Research Foundation (FWO Vlaanderen) for a (post) doctoral fellowship.

\section{References}

[1] Jonas Hansryd, Peter A. Andrekson, Mathias Westlund, Jie Li, and Per-Olof Hedekvist,” Fiber-Based Optical Parametric Amplifiers and Their Applications”, IEEE Journal of Selected Topics in Quantum Electronics 8, 506-520 (2002).

[2] John D. Harvey, Rainer Leonhardt, Stéphane Coen, Gordon K. L. Wong, Jonathan C. Knight, William J. Wadsworth, and Philip St.J. Russell, “Scalar modulation instability in the normal dispersion regime by use of a photonic crystal fiber,” Opt. Lett. 28, $2225-2227$ (2003).

[3] Michael R. Lamont, Barry Luther-Davies, Duk-Yong Choi, Steve Madden, Xin Gai, and Benjamin J. Eggleton, "Net-gain from a parametric amplifier on a chalcogenide optical chip,” Opt. Express 16, 20374-20381 (2008).

[4] Mark A. Foster, Amy C. Turner, Jay E. Sharping, Bradley S. Schmidt, Michal Lipson and Alexander L. Gaeta, "Broad-band optical parametric gain on a silicon photonic chip,” Nature 441, 960-963 (2006).

[5] R. M. Osgood, Jr., N. C. Panoiu, J. I. Dadap, Xiaoping Liu, Xiaogang Chen, I-Wei Hsieh, E. Dulkeith, W. M. Green, and Y. A. Vlasov, "Engineering nonlinearities in nanoscale optical systems: physics and applications in dispersion-engineered silicon nanophotonic wires," Advances in Optics and Photonics 1, 162-235 (2009).

[6] Sanja Zlatanovic, Jung S. Park, Slaven Moro, Jose M. Chavez Boggio, Ivan B. Divliansky, Nikola Alic, Shayan Mookherjea, and Stojan Radic, "Mid-infrared wavelength conversion in silicon waveguides using ultracompact telecom-band-derived pump source," Nature Photonics 4, 561-564 (2010).

[7] Xiaoping Liu, Richard M. Osgood, Jr., Yurii A. Vlasov, and William M. J. Green, "Mid-infrared optical parametric amplifier using silicon nanophotonic waveguides,” Nature Photonics 4, 557-560 (2010).

[8] Richard A. Soref, Stephen J. Emelett, and Walter R. Buchwald, “Silicon waveguided components for the long-wave infrared region,” J. Opt. A: Pure Appl. Opt. 8, 840-848 (2006). 